\title{
Adquisición del conocimiento en procesos de innovación abiertos. \\ Una aplicación en el sector de las TIC en España.
}

María Guadalupe Calderón Martínez*

\section{RESUMEN}

El objetivo principal de este artículo es analizar la adquisición de conocimientos en procesos abiertos de innovación mediante el establecimiento de acuerdos de colaboración por parte de empresas. La cooperación puede orientarse hacia múltiples dimensiones de la actividad de una organización. En algunos casos, es motivada por la necesidad de responder a cambios en el entorno, aunque también ha mostrado ser indispensable por la complejidad en el desarrollo de nuevos productos y la necesidad de acceder al conocimiento que no se encuentra disponible dentro de las propias organizaciones. En el marco tecnológico, esta interacción se produce entre entidades que, simultáneamente, compiten y cooperan, en la generación de tecnología avanzada, así como en su incorporación a los procesos productivos y desarrollo de nuevos productos.

Número de clasificación: $\mathrm{O} 32$

Palabras clave: innovación tecnológica, innovación abierta, acuerdos de colaboración.

\section{AbSTRACT}

The aim objective of this paper is to analyse the knowledge capture in open innovation, through the establishment of collaboration agreements in firms. The cooperation can be directed towards multiple dimensions of the activity of an organization. In some cases it is motivated by the need to respond to changes in the environment or due to the complexity in the development of new products and the need to knowledge access. Technological interaction occurs in entities that compete and cooperate in the creation of advanced technology, as well as the incorporation into production processes and new product development.

Clasification number: 032

Key words: technological innovation, open innovation, collaboration agreements.

* Profesora de asignatura en la Facultad de Estudios Superiores Cuautitlán, Universidad Nacional Autónoma de México. Alumna del Programa de Doctorado Interuniversitario en Economía y Gestión de la Innovación y Política Tecnológica, Universidad Complutense de Madrid. Correo electrónico:mgcm.unam@gmail.com. 


\section{INTRODUCCIÓN}

Una parte central en los procesos de innovación se refiere a la forma en que las empresas buscan encontrar nuevas ideas que puedan materializar y comercializar. En este contexto, las empresas innovadoras han adoptado estrategias que implican usar una amplia gama de actores y fuentes de conocimiento que les permitan alcanzar y mantener sus innovaciones (Schumpeter, 1942; Rosenberg, 1982; Von Hippel, 1986; Chesbrough, 2003). El concepto de procesos abiertos para la innovación se define como una estrategia en la forma de reorganizar, exteriorizar y mejorar la forma de innovar, mediante alianzas de colaboración con otras organizaciones, incluyendo en estos acuerdos, de forma particular, las actividades de ID $+\mathrm{i}$, la gestión de la propiedad intelectual-industrial, la búsqueda de fuentes externas de conocimiento, y los mecanismos opcionales de salida al mercado. El establecimiento de alianzas de colaboración con otras entidades se considera uno de los ejes centrales de los procesos abiertos, de ahí que nuestro objetivo sea analizar y describir la adquisición de conocimiento, resultado de una innovación conjunta.

Si bien en la revisión de la bibliografía hemos visto que los procesos abiertos conforman un modelo definido por la interacción de un gran número de variables, este artículo se centra en el análisis de la influencia de aquellos factores relacionados con el uso de fuentes externas de conocimiento y las características de los participantes en los acuerdos de colaboración.

Primero, describiremos los antecedentes, conceptos y características de los procesos de innovación, que nos permitirán dar paso al enfoque de los procesos abiertos y a la formulación de las hipótesis. En seguida, exponemos la metodología utilizada para la creación de la base de datos de nuestro estudio, para finalmente presentar el análisis descriptivo de los datos y exponer conclusiones previas que forman parte de la tesis doctoral sobre el valor estratégico de los acuerdos de colaboración para la adquisición de conocimiento en procesos abiertos de innovación. Posteriormente se procederá a identificar las futuras líneas de trabajo que nos lleven a continuar dicha investigación.

\section{Marco teórico}

Con el fin de situar el concepto de innovación es conveniente hacer una distinción entre invención e innovación. La invención es la primera idea para un nuevo producto o proceso, mientras que la innovación es el primer esfuerzo por lle- varlo a la práctica. Una innovación es la introducción de un producto - bien o servicio - o de un proceso, nuevo o significativamente mejorado, o la introducción de un método de comercialización o de organización nuevo aplicado a las prácticas de negocio, a la organización del trabajo o a las relaciones externas (OCDE, 2005b).

La tecnología en general, y el conocimiento en particular, han aumentado su complejidad, incrementando a su vez la importancia de las relaciones entre empresas y otras organizaciones para adquirir conocimiento especializado. Aunque la ID desempeña un papel vital en el proceso de innovación, buena parte de la actividad innovadora se sustenta en interacciones con otras empresas e instituciones públicas de investigación o en una estructura organizativa que fomente el aprendizaje y explote el conocimiento. Así, encontramos la adopción de diversas estrategias que favorecen la colaboración, desde la concentración territorial de diversas entidades con características particulares que contribuyen a la creación de un entorno innovador hasta el desarrollo conjunto por iniciativa de las propias organizaciones.

\section{INNOVACIÓN}

De las aportaciones iniciales de Schumpeter (1912) surgen los conceptos que se toman como punto de partida para establecer la diferencia entre invención, innovación y difusión. Se define una invención como aquel producto o proceso que ocurre en el ámbito científico-técnico y perdura en el mismo (ciencia pura o básica). Dicho autor relacionó la innovación con un cambio de índole económico y destacó la decisión del empresario de comercializar un invento como el paso decisivo para que éste conduzca a una innovación (Schumpeter, 1942); asimismo, definió al empresario como el innovador. Sin la existencia de emprendedores que lleven a cabo innovaciones, la tasa de crecimiento estaría limitada al aumento de los factores de la producción y se dificultaría la generación de dicho proceso. Toda esta evolución representa la destrucción creadora.

Durante las décadas de los setenta y los ochenta aparece el pensamiento evolucionista (o neoschumpeteriano), el cual incluye un conjunto heterogéneo de autores que han realizado otras aportaciones para definir la innovación. La idea que une estos enfoques es la concepción del desarrollo tecnológico como un proceso evolutivo, dinámico, acumulativo y sistémico.

Convencionalmente, la innovación se analiza como un proceso dividido en dos etapas: la primera consistiría en el desarrollo y la comercialización inicial 
de un nuevo producto o proceso, en tanto la segunda estaría dada por la aplicación generalizada, es decir, por la difusión de esa innovación. Bajo este análisis los problemas sólo pueden resolverse en la primera etapa, y la difusión es sencillamente la asimilación de la información técnica desarrollada en la etapa de innovación. En cambio, la visión neoschumpeteriana plantea que durante el proceso de difusión sigue teniendo lugar el progreso técnico inicialmente disparado por una innovación mayor.

Los autores evolucionistas consideran que la tecnología se desarrolla a la vez que se difunde, y lo hace en un contexto determinado, con ciertas características políticas, económicas, históricas e institucionales, con el cual se va dando un proceso de realimentación continua. Por ello, no toda la innovación generada por una empresa tendrá el mismo impacto; dependerá de la recepción de la misma en el entorno. Del mismo modo, la capacidad de innovar de una empresa estará influida por el medio que la rodea (Rozga, 1999). En este contexto se establece la importancia del ambiente en las actividades innovativas, ya que éstas dependen de las herramientas que el medio ofrezca para la toma de decisiones bajo incertidumbre, tales como avances científicos disponibles, soluciones aplicadas en otras empresas, redes de cooperación, entre otras. A su vez, la empresa se relaciona con diversas instituciones a partir de diferentes canales formales e informales, ya que dichas relaciones dan forma a sus actividades (Dosi y Malerba, 1996). Los actores del entorno y sus conexiones son influyentes en relación con la innovación y su difusión.

En los inicios de la década de los noventa se acentuó el desempeño de los factores particulares de una nación o región para promover el cambio tecnológico, la cual destaca el llamado aprendizaje por interacción, donde usuarios, proveedores, competidores y la infraestructura científica y tecnológica interactúan en la generación de innovaciones. En este sentido, surge la noción de sistema nacional de innovación (SNI) como un modelo interactivo de creación y uso del conocimiento en el cual participan los diferentes agentes relacionados con la producción y el desarrollo tecnológico, resultado de la necesidad de estudiar los procesos de innovación bajo una visión sistémica y de la importancia atribuida al contexto institucional y el factor organizacional en el aprendizaje y difusión de tecnologías (Cassiolato, 1994). El conjunto de aportaciones para la conformación del concepto de sistema nacional de innovación (Lundvall, 1992; Nelson, 1993; Freeman, 1995, y Metcalfe, 1995), muestran que la idea se basa en el supuesto de que los vínculos o relaciones entre los agentes involucrados en la innovación es un factor esencial para mejorar el desempeño tecnológico. En otras palabras, dado que la innovación y el progreso técnico son el resultado de una compleja serie de relaciones entre los agentes que producen, distribuyen y aplican el conocimiento, el desempeño innovador de un país dependerá en gran medida de cómo esos agentes se relacionen entre sí como partes o elementos integrantes de un sistema colectivo de generación de conocimientos.

La evolución en el concepto de innovación ha dado lugar a distintos modelos que tratan de explicar los procesos por los cuales esta actividad se lleva a cabo, generando a su vez cambios en la conformación de éstos. Es aquí hacia donde dirigimos la atención en esta parte de nuestro ámbito de estudio, es decir, hacia la influencia de los distintos factores que propician y dan lugar a la apertura de los procesos de innovación.

\section{Modelos DE INNOVACIÓN}

El proceso de innovación es algo complejo que integra instituciones, personas, actividades, estrategias y mecanismos de gestión. Teniendo en cuenta la relación causa-efecto existente entre innovación, competitividad y crecimiento, resulta fundamental disponer de ciertos procesos que permitan alcanzar un elevado desempeño en materia de innovación. A partir de los años cincuenta surgen una serie de modelos para explicar el proceso de innovación, de los cuales mencionamos aquellos que han llevado a un cambio en la forma de explicar la innovación, que incorporan diversos agentes y elementos organizativos que facilitan la apertura hacia otras empresas e instituciones.

El cuadro 1 recoge distintos modelos que han explicado el proceso de innovación, y que de acuerdo con la revisión de la bibliografía, han dado lugar a una evolución en las características que definen dichos procesos. En esta primera descripción tomamos en cuenta únicamente un enfoque cerrado; los primeros modelos utilizados para describir el proceso de innovación se basaban en la premisa de que el cambio técnico es el resultado de la investigación básica, de la cual se deriva la posibilidad de realizar investigación aplicada. Esta última posibilita a su vez la realización de desarrollos experimentales que dan lugar a la innovación tecnológica y a su difusión. Mientras más se invierta en investigación básica y aplicada, mejores serán los resultados alcanzados.

En estos modelos, la innovación tecnológica es descrita como un proceso en el que unos inputs se convierten en productos a lo largo de una serie de pasos. Así, los primeros modelos sobre el proceso de innovación no dejan de tener un valor, ya que establecieron las bases para construir conceptos posteriores. 
Cuadro 1. Evolución en los modelos de innovación

\begin{tabular}{|c|c|c|}
\hline \multicolumn{3}{|c|}{ PROCESOS CERRADOS } \\
\hline \multicolumn{3}{|c|}{ Modelos lineales } \\
\hline Technology Push & Schumpeter (1934) & $\begin{array}{l}\text { - La innovación parte desde la ciencia hacia la } \\
\text { tecnología. }\end{array}$ \\
\hline Market Pull & Schmookler (1962) & $\begin{array}{l}\text { - Las necesidades de los consumidores se } \\
\text { convierten en la principal fuente de ideas. }\end{array}$ \\
\hline \multicolumn{3}{|c|}{ Modelos interactivos } \\
\hline Coupling Model & $\begin{array}{l}\text { Mowery y Rosenberg } \\
\text { (1978) }\end{array}$ & $\begin{array}{l}\text { - Confluencia entre capacidades tecnológicas } \\
\text { y necesidades del mercado. }\end{array}$ \\
\hline Chain Link & Kline y Rosenberg (1986) & $\begin{array}{l}\text { - Sistema multidimensional asocia la } \\
\text { integración interna y externa. } \\
\text { - Relaciona ciencia y tecnología en todas las } \\
\text { etapas del proceso }\end{array}$ \\
\hline \multicolumn{3}{|c|}{ Modelos integrados } \\
\hline $\begin{array}{l}\text { System Integration o } \\
\text { Parallel Development }\end{array}$ & $\begin{array}{l}\text { Graves (1987) } \\
\text { Rothwell (1992) }\end{array}$ & $\begin{array}{l}\text { - Considera el tiempo como una variable } \\
\text { crítica del proceso. } \\
\text { - Fases no secuenciales, procesos traslapados, } \\
\text { concurrentes o simultáneos. }\end{array}$ \\
\hline
\end{tabular}

Fuente: Elaboración propia con información y asesoría proporcionada por Patricio Morcillo, catedrático de la Universidad Autónoma de Madrid.

\section{Innovación abierta}

No podemos considerar los procesos de innovación como un planteamiento endógeno y estático porque la empresa es un espacio social que se relaciona permanentemente con el entorno. Por tanto, la supervivencia y crecimiento de cualquier compañía depende en gran medida de su adaptación continua al medio, del dominio de su cartera de competencias y, por tanto, la renovación de la misma que dependerá de su capacidad de aprendizaje.

Desde esta perspectiva, resulta interesante que las empresas cooperen y den valor en sus planteamientos organizativos a la dimensión externa porque la cultura en general, y la cultura empresarial en particular, es el aprovechamiento organizacional y social del conocimiento (Morcillo, 2006). Las fuentes externas complementan los conocimientos de la organización. Se darán situaciones en las
Cuadro 2. Evolución en los modelos de innovación

PROCESOS ABIERTOS

\begin{tabular}{|c|c|c|}
\hline \multicolumn{3}{|c|}{ Modelos de carácter estratégico } \\
\hline $\begin{array}{l}\text { Redes de } \\
\text { colaboración. }\end{array}$ & $\begin{array}{l}\text { Saxenian (1994), } \\
\text { Castells (1996), } \\
\text { Shapiro y Varian } \\
\text { (1998). }\end{array}$ & $\begin{array}{l}\text { - Organizaciones con cierto grado de } \\
\text { interdependencia, que innovan en una gama } \\
\text { definida de productos o servicios. }\end{array}$ \\
\hline $\begin{array}{l}\text { Sistema nacional de } \\
\text { innovación. }\end{array}$ & $\begin{array}{l}\text { Lundvall (1992), } \\
\text { Freeman (1993), } \\
\text { Metcalfe (1995) }\end{array}$ & $\begin{array}{l}\text { - Sistema interactivo de empresas privadas y } \\
\text { públicas, universidades y agencias estatales, } \\
\text { para producir ciencia y tecnología dentro de las } \\
\text { fronteras nacionales. }\end{array}$ \\
\hline Triple hélice & $\begin{array}{l}\text { Leysderdoff y Ezkowitz } \\
\text { (1995) }\end{array}$ & $\begin{array}{l}\text { - Interacción de universidades como productores } \\
\text { de conocimiento, empresas como creadoras } \\
\text { de negocios y la administración pública como } \\
\text { regulador y generador de entornos. }\end{array}$ \\
\hline \multicolumn{3}{|c|}{ Modelos de carácter organizativo } \\
\hline $\begin{array}{l}\text { Iniciativas internas de } \\
\text { cooperación. }\end{array}$ & $\begin{array}{l}\text { Mintzberg (1983), } \\
\text { Handy (1990), } \\
\text { Nonaka y Takeuchi } \\
\text { (1995) }\end{array}$ & $\begin{array}{l}\text { - Motivación del grupo de proyecto, aprendizaje, } \\
\text { fluidez en la comunicación, clima de confianza } \\
\text { dentro y hacia los agentes externos. }\end{array}$ \\
\hline $\begin{array}{l}\text { Iniciativas externas de } \\
\text { cooperación. }\end{array}$ & $\begin{array}{l}\text { Von Hippel (1986), } \\
\text { Chesbrough (2003), } \\
\text { Leadbeater (2006) }\end{array}$ & $\begin{array}{l}\text { - Intercambio de conocimiento, difusión y } \\
\text { transferencia de tecnología. Las fuentes } \\
\text { externas complementan el conocimiento } \\
\text { interno. Movilización de agentes y recursos. } \\
\text { Dominio de la cartera de competencias } \\
\text { esenciales. }\end{array}$ \\
\hline
\end{tabular}

Fuente: Elaboración propia con información y asesoría proporcionada por Patricio Morcillo, catedrático de la Universidad Autónoma de Madrid.

que a la empresa le interese explotar su conocimiento junto con el de las fuentes externas para poder innovar. Ahora es claro que en el caso de la cooperación externa será imprescindible fijar claros controles de gestión para evitar la transmisión de capacidades o habilidades básicas sobre las que descansan las competencias esenciales de la empresa. Pero, al margen de los procesos de aprendizaje que se desarrollan de manera conjunta, es importante contar con ciertas infraestructuras que faciliten la ejecución de los acuerdos, la transferencia de tecnología y la protección de las innovaciones. 
El concepto de procesos abiertos se entiende como aquello que se concibe admitiendo y explotando los cambios surgidos en el entorno y que aprovecha la porosidad de las fronteras de la empresa (Chesbrough, 2003). Al igual que en el enfoque cerrado, han surgido distintos modelos de acuerdo con las características que los definen (cuadro 2). En este tipo de procesos, el conocimiento externo desempeña un papel de igual a igual en proporción con los conocimientos internos. Esto hace que la empresa tenga la responsabilidad de instaurar los mecanismos necesarios que faciliten su búsqueda e incorporación en los procesos de trabajo y producción.

Los procesos abiertos para la innovación constituyen una estrategia, opcional a la integración vertical, que busca la creación de valor y en la que algunas empresas necesitan identificar el conocimiento externo e incorporarlo, mientras que otras buscan mercados para sus innovaciones (West y Gallager, 2006). Estudios previos han demostrado la función de los lazos interorganizacionales en la innovación, es decir, la cooperación entre empresas incrementa el potencial de conocimiento y reduce la duplicidad de esfuerzos (Teece, 1989), además se sabe que las entidades integradas en diferentes tipos de alianzas son más innovadoras que aquellas que sólo se involucran en una clase de acuerdo por la variedad de conocimiento que se puede obtener a partir de esta interacción (Powell, et al., 1999). La figura 1 representa el modelo abierto; en éste, los proyectos pueden ser impulsados desde fuentes internas o externas, al mismo tiempo que una nueva tecnología puede entrar al proceso en cualquier etapa.

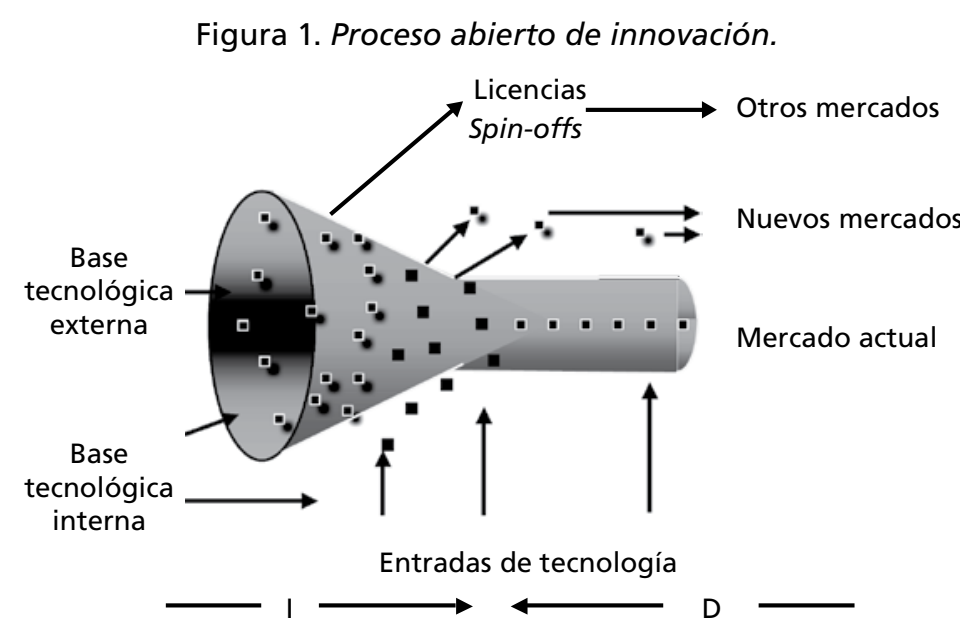

Fuente: Chesbrough (2006).
Por medio de este esquema vemos cómo en el caso de los procesos abiertos, las ideas, información y conocimiento no sólo provienen del interior de la organización, sino también del exterior. Además, los proyectos pueden salir de varias formas, bien a partir de licencias o alcanzar el mercado por los canales de la compañía. En cuanto a los resultados obtenidos, al tratarse de un proceso colaborativo, no existe una única salida sino múltiples outputs que pueden ser utilizados, mediante acuerdos, por los distintos agentes que han participado en el proceso. Otra característica de los procesos abiertos es que sus beneficios pueden conseguirse por medio de clusters regionales, considerando que el efecto de las redes en la innovación se magnifica con la proximidad geográfica (Porter, 1998). Podemos resumir los puntos significativos que definen los procesos abiertos:

- Se concede la misma importancia al conocimiento interno que al que pueda provenir del exterior.

- Se reconoce la importancia de las personas en la incorporación del conocimiento, ya sea que éstas pertenezcan o no a la empresa.

- El objeto principal de este modelo es generar valor a través de los resultados de la ID $+\mathrm{i}$.

- Se propicia la existencia de flujos tecnológicos y de conocimiento.

- Existe un incremento en el número de intermediarios en los procesos de innovación.

- Aparecen nuevos indicadores para calcular la capacidad de innovación y el desempeño.

- Se favorece el uso de la propiedad intelectual-industrial como fuente de información, así como su adquisición, toda vez que actúa como mecanismo de protección de la tecnología.

Ahora bien, las empresas pueden captar y crear valor a partir de las nuevas ideas de maneras distintas: incorporando la tecnología a su quehacer actual, concediendo licencias o emprendiendo nuevas alianzas para explotar su tecnología en un nuevo ámbito, etcétera. Un aspecto crítico en este proceso es que la tecnología por sí misma no tiene un valor objetivo; el valor económico permanece latente hasta que se comercializa de alguna forma y se obtienen retornos o beneficios.

En esta parte del estudio empírico, nuestro objetivo es analizar y describir la adquisición de conocimientos resultado de una innovación conjunta. Para ello hemos seleccionado como fuente de datos las patentes registradas por em- 
presas españolas en el sector de las TIC; en la metodología se explicarán los pasos para la obtención de los registros seleccionados.

\section{CONTEXTO DEL SECTOR DE LAS TIC EN ESPAÑA}

El interés por este sector, además de sus características tecnológicas, surge por el impacto económico que representa. Los sectores que hacen un uso intenso de las TIC son los de la fabricación, la automoción, la industria aeroespacial, la industria farmacéutica, la maquinaria médica y la industria agroalimentaria, sin olvidar los servicios financieros, los medios de comunicación y la venta al detalle (COTEC, 2000). El uso de las TIC ha redundado en diversos beneficios para las empresas: desarrollo más rápido de productos, reducciones de costos y gastos indirectos, transacciones más rápidas y fiables, una mejor relación con clientes y proveedores, niveles más altos de servicio y apoyo al cliente, y también mejores oportunidades de colaboración.

Por otra parte, en especial para el caso de España, el periodo seleccionado permite analizar el lapso posterior a la apertura definitiva del sector y situar su contexto hasta 2007. Dicho año marca, además, el comienzo de un ciclo clave para el desarrollo tecnológico de las empresas europeas y españolas por el inicio del VII Programa Marco que dentro del programa Cooperación ubica las tecnologías de la información y la comunicación como área preponderante. Integrado en las perspectivas financieras de la Unión Europea, determinará la actividad y las políticas comunitarias de ID + i hasta el año 2013. El análisis del impacto de este conjunto de políticas comprendidas bajo el Programa Marco podría constituir un tema de investigación en forma comparativa respecto a nuestro análisis actual.

El sector de las telecomunicaciones ha experimentado un importante cambio estructural durante los años recientes en cuanto a los aspectos regulatorios y de mercado. El motivo, sin duda, está en los cambios, económico y tecnológico, que han permitido la liberalización del sector en España y su participación global en los mercados. La conjunción de las mejoras tecnológicas y el aumento de la demanda de servicios rompieron los cimientos de los monopolios e hicieron patente la necesidad de atender más de cerca las necesidades de consumidores y de la sociedad en general (ASIMELEC, 2009). Como resultado del proceso de innovación, las TIC aumentan y transforman la creación y la aplicación económica del conocimiento (Villaseca y Torrent, 2008). Sin embargo, se requiere la entrada constante de ideas que circulen en las redes de conocimiento, que permitan soportar una oferta de nuevos y mejores servicios, que con su utilización posibi- liten la creación de nuevos hábitos que deriven en necesidades más sofisticadas en el mercado. Esto nos lleva entonces a considerar otros factores fundamentales y condicionantes del desarrollo de las TIC y, por tanto, de la economía de la información: el establecimiento de mecanismos de colaboración y su correspondiente regulación.

\section{Hipótesis}

Los innovadores rara vez llevan a cabo esta actividad de manera aislada en lugar de ello, tienden a unirse en coaliciones (Laursen y Salter, 2006) anidadas en comunidades innovadoras que se relacionan mediante una red de interacciones (Scott y Brown, 1999). Para tener acceso a estas redes y fuentes externas, las empresas necesitan contar con la capacidad para absorber las ideas provenientes de fuentes externas e integrarlas con sus procesos internos a fin de lograr una innovación (Cohen y Levinthal, 1990). Si bien nuestra investigación pretende dar cuenta, posteriormente, sobre la estrategia de una organización para captar o apropiarse de los beneficios de sus actividades innovadoras (Teece, 1986; Chesbrough, 2003 y 2006), en este artículo se desea destacar el uso de mecanismos formales que permiten a las organizaciones el intercambio de conocimientos con su entorno.

Teniendo en cuenta que nuestro objetivo en esta parte del estudio empírico es el análisis sobre el establecimiento de acuerdos de colaboración en procesos abiertos de innovación para la adquisición del conocimiento, planteamos las siguientes hipótesis a contrastar:

- Hipótesis 1: La elección de un socio en los procesos de innovación influye en el establecimiento de algún acuerdo de cooperación.

- Hipótesis 2: La nacionalidad de los inventores que intervienen en los procesos de innovación influye en el establecimiento de algún acuerdo de cooperación.

- Hipótesis 3: La incorporación de patentes como fuente externa de conocimientos para la innovación, tiene que ver con la participación en acuerdos de cooperación.

- Hipótesis 4: La incorporación de patentes extranjeras como fuente externa de conocimientos para la innovación, determina el establecimiento de acuerdos de cooperación. 
- Hipótesis 5: El uso de artículos científicos, como fuente externa de conocimientos para la innovación, influye en el establecimiento de acuerdos de cooperación.

Existen diversos trabajos que han descrito las tendencias en el uso de los acuerdos de colaboración por parte de empresas españolas (Menguzzato, 1992; García, 1992; Cassiman, 1999; Álvarez et al., 2005). Partiendo de bases de datos construidas con diversas metodologías, en general se observan ciertas tendencias hacia el predominio de los acuerdos internacionales. Dentro de ellos, el mayor número de acuerdos es con empresas europeas. También se observa que existe un gran peso de los acuerdos suscritos por dos socios.

Para nuestro estudio hemos decido utilizar la información contenida en las patentes, por ser un indicador del cual podemos extraer distintos datos. Su uso como fuente de información tecnológica resulta de especial aplicación al establecer la frontera tecnológica o del estado del arte, básico en la etapa de definición de los proyectos tecnológicos. Es aquí donde la empresa tendrá que gestionar sus procesos de exploración de las innovaciones existentes, incluyendo la búsqueda de nuevas ideas para el desarrollo de productos, nuevas formas de organización o soluciones a problemas existentes (Stuart y Podolny, 1996; Koput, 1997; Katila, 2002; Katila y Ahuja, 2002), y por supuesto el aprendizaje organizacional. Respecto a los datos de patentes, éstos pueden ser analizados y agregados de varias maneras, inclusive se pueden combinar, según el objetivo de las necesidades de investigación, abordando distintos enfoques, siempre con prudencia, en la utilización e interpretación de los resultados (OCDE, 1994).

En el caso de las empresas españolas, se concede gran importancia a las patentes como instrumento de defensa de la propiedad industrial y en menor medida como instrumento que proporcione mejor capacidad de negociación. Tan sólo el segmento de grandes empresas considera importantes las patentes como instrumento de ayuda en la exportación de la tecnología y formalización del conocimiento. Sin embargo, se menciona como principal finalidad realizar búsquedas previas al comienzo de un proyecto de ID en la empresa, evitando invertir en algo ya existente, y para obtener información acerca de los competidores (Hidalgo, 2003). En las patentes, es posible encontrar, además de la información técnica, una serie de datos que sobrepasan ese ámbito, permitiendo que su uso no se restrinja al aspecto técnico, en campos como el código de clasificación, el solicitante, el inventor y dentro del informe sobre el estado de la técnica. Esto multiplica las opciones de uso de la información; tales cuestiones pueden referirse al origen de una tecnología, al seguimiento de las actividades innovadoras de una determinada empresa o sector, etcétera.

\section{Metodología de LA INVESTigaCióN}

Teniendo presente que el objetivo del estudio es establecer un contraste sobre los procesos abiertos para la innovación en el sector de las TIC en España, a partir del análisis de patentes durante el periodo de 1999 al 2007, el primer paso fue diseñar una base de datos propia, aplicando la siguiente metodología:

Se tomaron en cuenta las recomendaciones de la OCDE (1994), que hacen referencia a la necesidad de utilizar datos procedentes de oficinas de prestigio y con homogeneidad histórica en la forma de protección.

Para la realización del estudio se emplearon datos procedentes de la Oficina Europea de Patentes (EPO), utilizando la plataforma de búsqueda esp@cenet.

a) Se incluyeron las solicitudes de patentes con número de prioridad española, lo que implica la extensión del colectivo a empresas extranjeras que protegen sus invenciones en España, bien por estrategia de protección o porque la invención ha sido realizada en el país.

b) Se seleccionaron aquellos registros donde al menos uno de los solicitantes de la patente fuera una empresa española.

c) Se han excluido las solicitudes presentadas por el propio inventor, dado que este trabajo de investigación se enfoca en empresas que hayan patentado sus innovaciones en cualquiera de los sectores que integran la industria de las TIC.

d) Se seleccionaron aquellas patentes publicadas, cuyo informe sobre el estado de la técnica ha sido favorable, lo que implica que no contienen antecedentes que afecten la novedad o actividad inventiva. Por consiguiente, este colectivo se encuentra configurado por el conjunto de solicitudes de patentes que tienen un grado de calidad (Molero e Hidalgo, 2003).

f) Se incluyeron en la base de datos propia no sólo la información básica de las solicitudes de patentes (fechas, solicitantes, organismos, etcétera), sino que se han incluido las referencias de patentes y otros documentos citados en el informe sobre el estado de la técnica de cada una de ellas.

g) Para efectuar la búsqueda se utilizó la correspondencia de la clasificación internacional de patentes (CIP), con base en los códigos del Compendio de Estadísticas sobre Patentes 2005 (OCDE, 2005a). 
No obstante, los resultados obtenidos deben ser matizados, ya que el uso de patentes como fuente de conocimiento lleva asociado un conjunto de dificultades, como la existencia del secreto industrial y el hecho de que en determinados sectores el ciclo de vida de los productos es muy corto, por lo que no resulta de interés patentar. Pero a pesar de estas dificultades, la decisión de utilizar las solicitudes de patentes resulta válido por representar la culminación de un esfuerzo, en este caso por parte de varias organizaciones que emprendieron de manera conjunta la actividad innovadora, poniendo de relieve no sólo la intensidad de su actividad inventiva, sino también su capacidad para implantar sus competencias tecnológicas en la apertura de las propias fronteras organizacionales.

\section{ANÁLISIS DESCRIPTIVO}

La asignación de códigos CIP ha permitido ubicar 18 diferentes grupos dentro del sector de las telecomunicaciones: 8 para la electrónica de consumo, 13 para los ordenadores y equipo de oficina y 22 para los demás bienes ("otros"). En relación con la información contenida en los documentos de patente, seleccionamos los datos que aparecen en la figura 2, como aquellos que proporcionarán las referencias sobre las fuentes de conocimiento a las que una organización recurre en sus procesos de innovación.

Decidimos analizar en primer lugar la presencia de acuerdos de cooperación; la figura 3 representa los porcentajes de las empresas que cooperan y las que no lo hacen. Para ello, seleccionamos aquellas patentes que han sido registradas por más de un solo solicitante, con lo que asumimos que existe un acuerdo de colaboración de manera formal.

Si bien observamos que el porcentaje total de las empresas que cooperan es menor que el de aquellas que no lo hacen, en un gráfico posterior (figura 4) hemos agregado la variable tiempo para observar cómo ha sido su evolución a lo largo del periodo de estudio.

Tomando como base la variable "cooperación" podemos detectar diferencias entre las estrategias que han sido desarrolladas para la adquisición de conocimiento en los procesos de innovación. Empezaremos por hacer un análisis descriptivo sobre la presencia o no de mecanismos de colaboración y, en este caso, observar las características de los participantes en el proceso de innovación. En primer lugar el número total de patentes registradas en el sector ha ido aumentando a lo largo del tiempo. En segundo, si bien el número de patentes registradas por más de un solicitante continúa siendo menor al de aquellas en las
Figura 2. Datos extraídos de las patentes

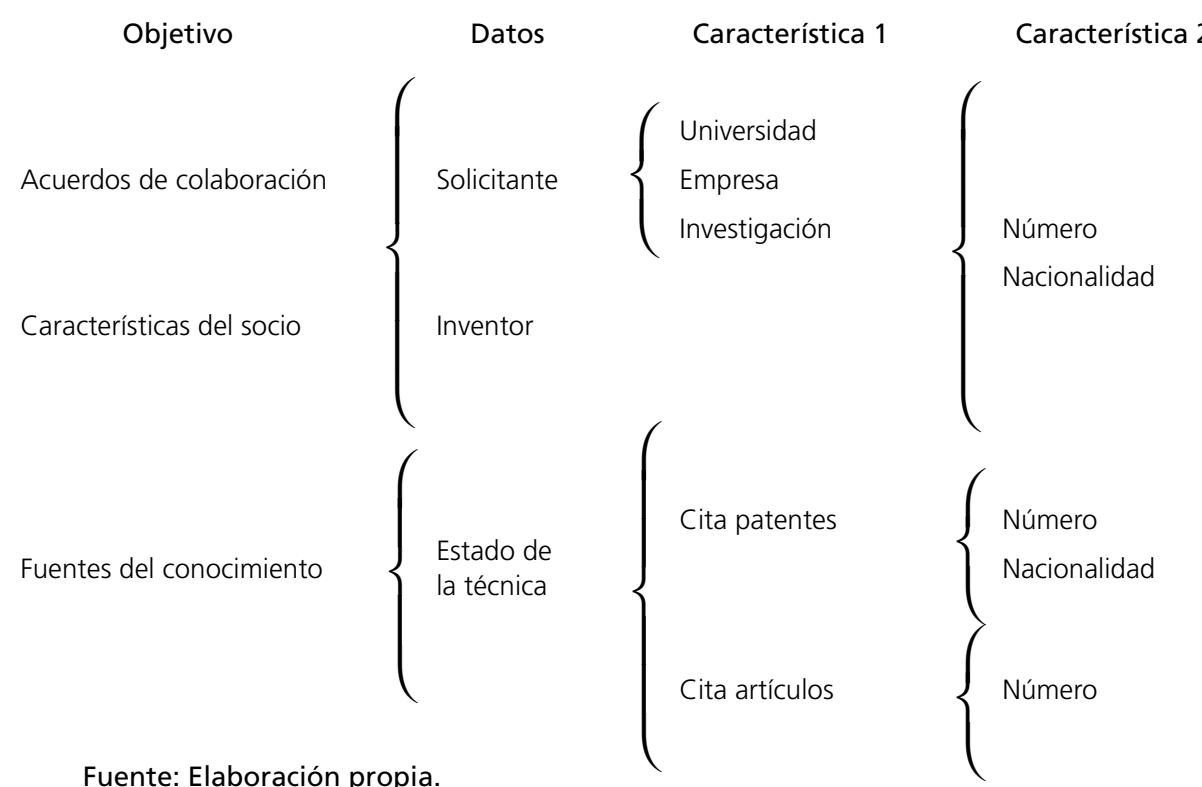

Figura 3. Establecimiento de acuerdos de cooperación

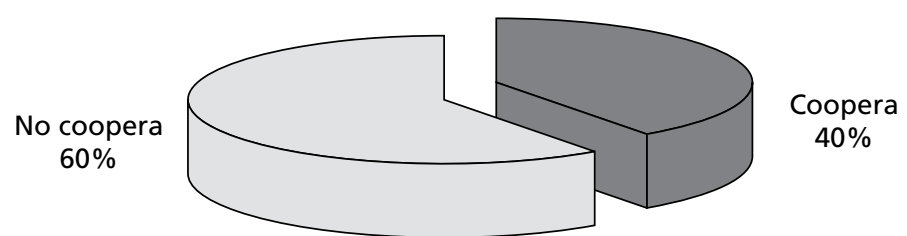

Fuente: Elaboración propia con información de la base de datos esp@cenet (2009).

que no ha habido un acuerdo de cooperación, al menos de manera formal encontramos que este último mantiene un crecimiento constante, mientras que el número de patentes en las que aparece más de un socio ha ido creciendo de forma más acelerada, acortando la brecha respecto a las empresas que llevan a cabo el proceso sin la incorporación de algún socio.

Otra característica a describir es la naturaleza de los participantes; entre aquellas empresas que han establecido acuerdos de cooperación encontramos que los socios más frecuentes son las universidades, seguidos en la misma proporción por las empresas e instituciones de investigación, pero no existe una dife- 
Figura 4. Establecimiento de acuerdos de cooperación en el periodo de estudio

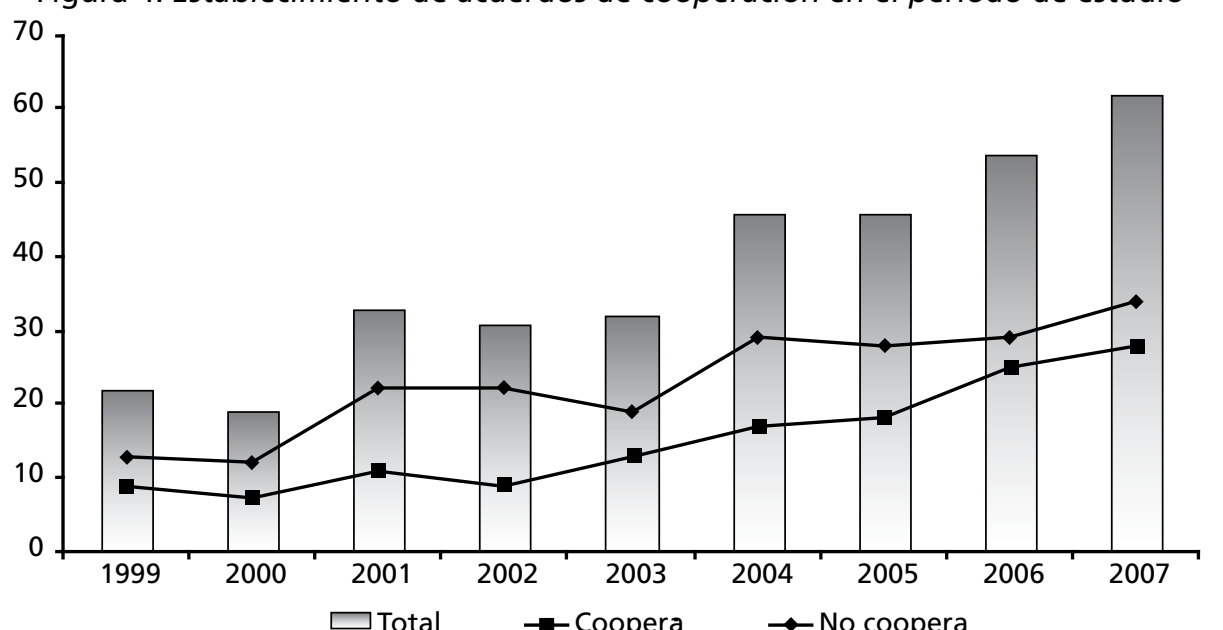

Fuente: Elaboración propia con información de la base de datos esp@cenet (2009).

rencia significativa entre estos datos que permita extraer conclusiones (figura 5). Podemos corroborar los porcentajes correspondientes, que nos dan información descriptiva, sin embargo, para realizar un estudio exploratorio sería necesario acudir a fuentes primarias de datos.

En la evolución temporal, el aumento en el registro de patentes en las que aparece más de un solicitante es congruente con la implantación de nuevos enfoques en los instrumentos y políticas de investigación e innovación (León, 2007), haciendo más explícita la influencia de la Unión Europea en el comportamiento dinámico del sistema ciencia, tecnología y empresa, y propiciando la interacción entre los diferentes actores que integran el sistema de innovación. De hecho, los gobiernos de países avanzados y múltiples grupos de expertos han

Figura 5. Elección de los socios en el establecimiento de acuerdos de cooperación

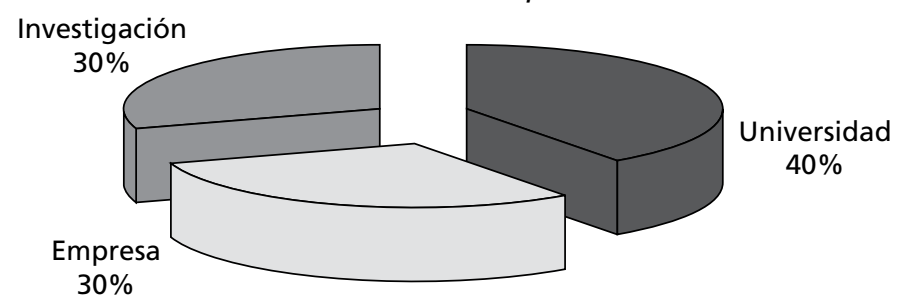

Fuente: Elaboración propia con información de la base de datos esp@cenet (2009). señalado que uno de los elementos clave para la construcción de una sociedad basada en el conocimiento competitiva en el ámbito internacional, reside en acelerar el carácter innovador de la sociedad para mantener una competitividad sostenible en el tiempo mediante la interacción de todos los actores en las actividades de ID + i (Aho et al., 2006; Etzkowitz y Leysderdoff, 1995; Etzkowitz et al., 2000; Foray, 2004).

Otro de los elementos que constituye un medio de obtención de conocimientos entre los participantes en los procesos de innovación son los inventores. En la figura 6 observamos la proporción de inventores nacionales y extranjeros que aparecen en las patentes. La participación de inventores nacionales es similar entre las empresas que cooperan y las que no cooperan, sin embargo, podemos observar una diferencia en la presencia de inventores extranjeros, siendo mayor en proporción en aquellas patentes donde sí hubo un acuerdo de cooperación. Es importante señalar que el acceso a fuentes externas para la obtención de conocimientos va más allá de las universidades y las instituciones de investigación, y abarca también a las pequeñas empresas especializadas, a los inventores e incluso al personal técnico.

Con el fin de profundizar en los mecanismos de adquisición del conocimiento, tendremos en cuenta el análisis de las patentes que aparecen citadas dentro del informe sobre el estado de la técnica de cada uno de los registros extraídos. En esta parte, respecto a los datos que se refieren al número total de citas, así como al país de origen de las mismas. Primero, en cuanto a su uso, hemos encontrado que $75 \%$ hace referencia a otras patentes, contra $25 \%$ que no reporta esta información en dicho informe (figura 7).

Figura 6. Nacionalidad de los inventores en acuerdos de cooperación

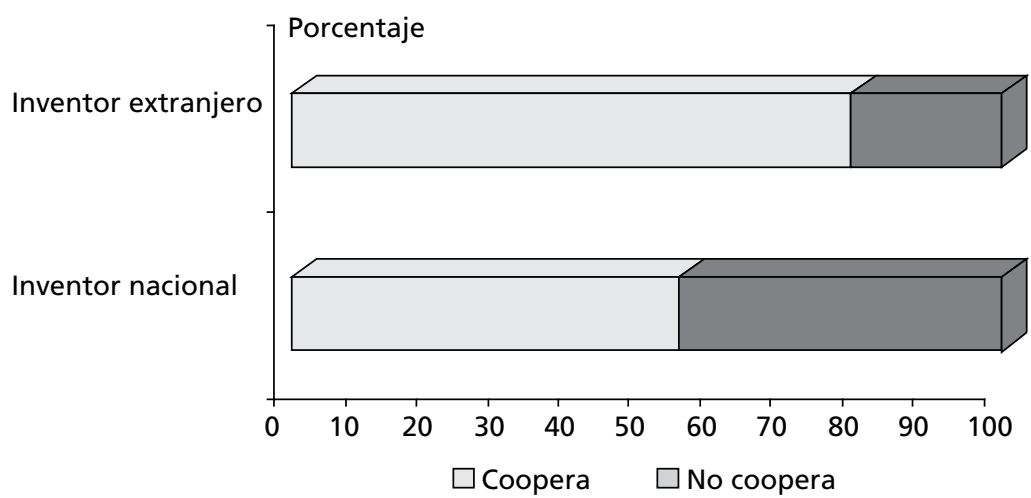

Fuente: Elaboración propia con información de la base de datos esp@cenet (2009). 
Figura 7. Cita de patentes en el informe sobre el estado de la técnica.

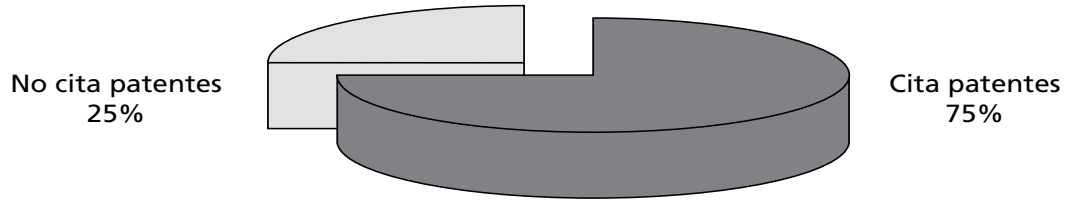

Fuente: Elaboración propia con información de la base de datos esp@cenet (2009).

Uno de nuestros primeros estudios nos llevó a encontrar que en las patentes registradas dentro del sector de las TIC se observa la tendencia a que las fuentes de conocimiento externo provengan de los bloques que integran el fenómeno de triadización (Ohmae, 1991), como muestra la figura 8. Si bien es mínima la aparición de patentes de otros países, consideramos oportuno mencionar que se encontraron estos registros en los datos a partir del año 2004, por lo que sería interesante analizar si el número de patentes que proceden de países que no pertenecen a la triada podría aumentar al tomarse en cuenta un lapso posterior. Si bien se observa un incremento en el total de patentes citadas, también es cierto que la tendencia a que estas provengan de Estados Unidos, Europa y Japón se mantiene constante en todo el lapso transcurrido.

En cuanto al uso de citas de patentes, se puede ver en la figura 9 que las empresas que establecen acuerdos de colaboración utilizan citas de otras patentes en más de $90 \%$ de los casos, lo que implica casi la totalidad de la muestra, pero no sucede así en los casos en que sólo aparece un titular de la patente, donde el uso de estas citas es superior a $60 \%$, en los casos obtenidos. Esto demuestra que las empresas que cooperan dan mayor importancia a la cita de patentes, lo cual puede ser un indicador de complejidad y de elementos como la novedad u originalidad de la innovación, que se relacionan con la calidad de una patente en función de los requisitos de patentabilidad (novedad, actividad inventiva y aplicabilidad industrial).

En cuanto a las citas, cabe distinguir dos tipos de documentos: las citas de otras patentes y las citas de otro tipo de documentos, entre los que se encuentra la bibliografía científica. Al igual que las citas de patentes, uno de los indicadores de la complejidad de la innovación lo constituyen las citas de otros documentos científicos (Guellec y Van Pottelsberghe, 2000). En nuestro análisis hemos seleccionado las citas de artículos científicos que aparecen en el informe sobre el estado de la técnica, que en comparación con las citas de patentes resulta un número inferior como lo muestra la figura 10 ; significa únicamente $17 \%$ del universo estudiado
Figura 8. Cita de patentes en el informe sobre el estado de la técnica por país y año

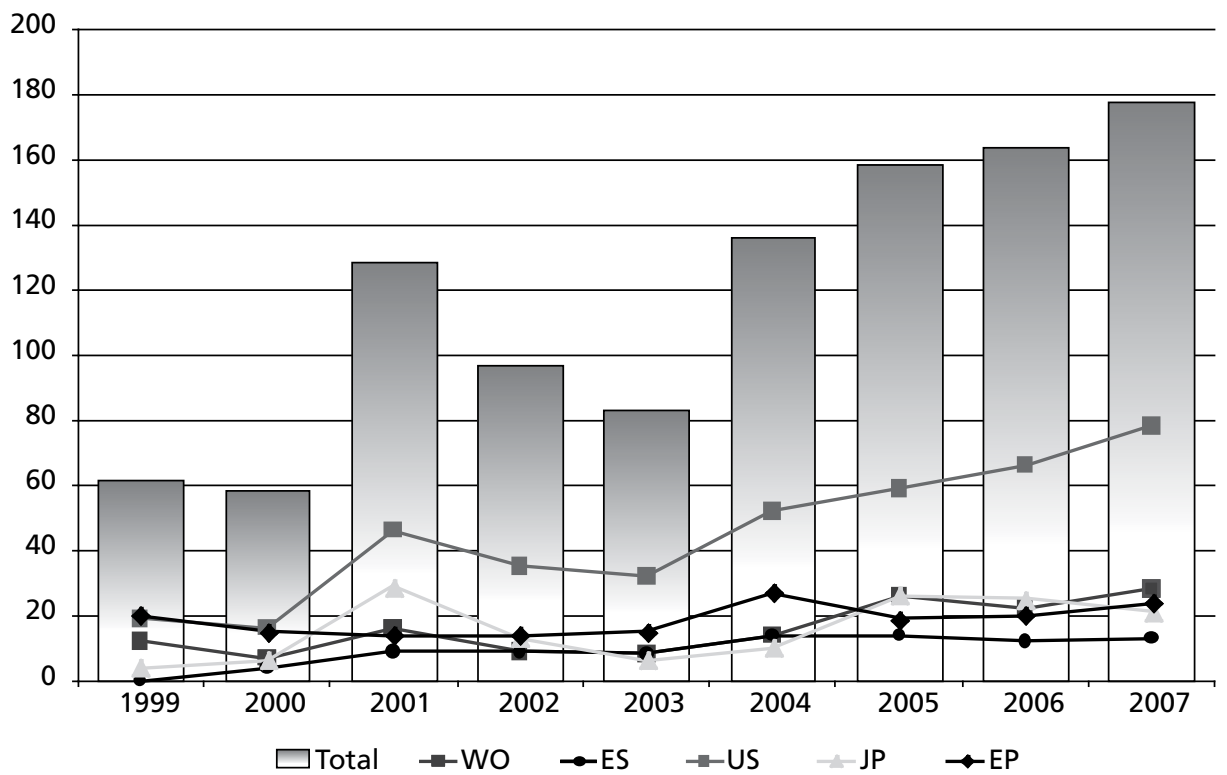

Fuente: Elaboración propia con información de la base de datos esp@cenet (2009).

Figura 9. Cita de patentes en el informe sobre el estado de la técnica en acuerdos de colaboración

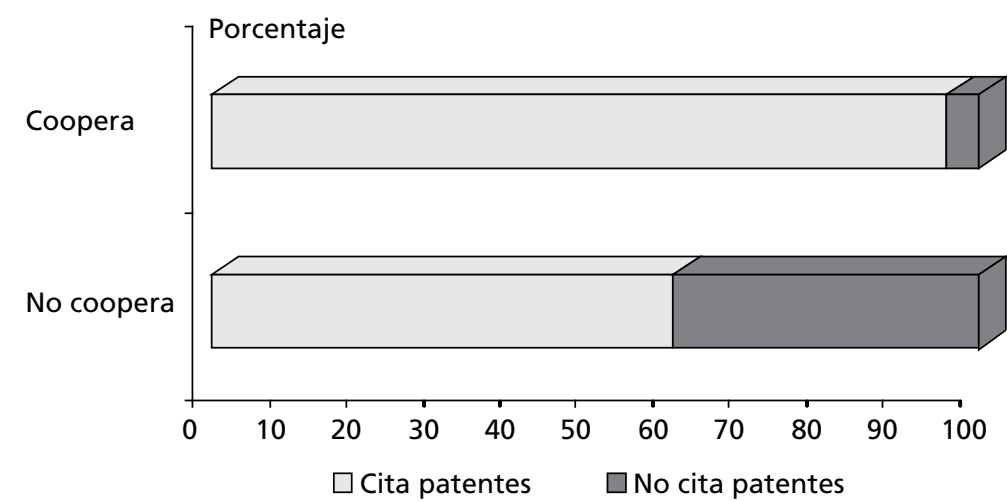

Fuente: Elaboración propia con información de la base de datos esp@cenet (2009). 
Es importante hacer notar que existen notorias diferencias entre las patentes registradas en la UGS Patent and Trademark Office (USPTO) y en la European Patent Office (EPO) en cuanto a las citas recogidas en las mismas, debido a las diferentes políticas empleadas. En los Estados Unidos no existen unas normas tan restrictivas como en Europa a la hora de exigir al solicitante de una patente no haber hecho público previamente, bajo ningún medio o procedimiento de comunicación, los descubrimientos para los que se solicita protección y, además, citar toda la información relevante para la patente. Por este motivo, las patentes registradas en la USPTO muestran, por lo general, un mayor número de citas y una mayor diversidad de conexiones temáticas que vinculan los campos del conocimiento. No obstante, es necesario destacar que en algunos casos la información sobre el estado de la técnica que aportan los informes tecnológicos podría resultar incompleta, ya que existe la posibilidad de no localizar algún documento significativo al momento de realizar la búsqueda documental.

Partiendo del hecho de que el conocimiento en sus más variadas formas ocupa un lugar primordial, puede afirmarse que estamos en una economía del aprendizaje (Molero e Hidalgo, 2003), en la que las organizaciones deben desarrollar formas de creación de nuevas bases de conocimiento que permitan acceder de manera eficiente al que generan terceros agentes. Hoy en día puede identificarse la existencia de procesos abiertos para la innovación a partir de diferentes estrategias o modelos de negocio en las organizaciones; éstos no son incompatibles y pueden coexistir en una misma organización. Podría ser objeto de un análisis posterior ubicar en las empresas innovadoras una serie de características que permitan proponer una clasificación que agrupe las distintas estrategias que se han detectado en este tipo de procesos.

Figura 10. Cita de artículos en el informe sobre el estado de la técnica

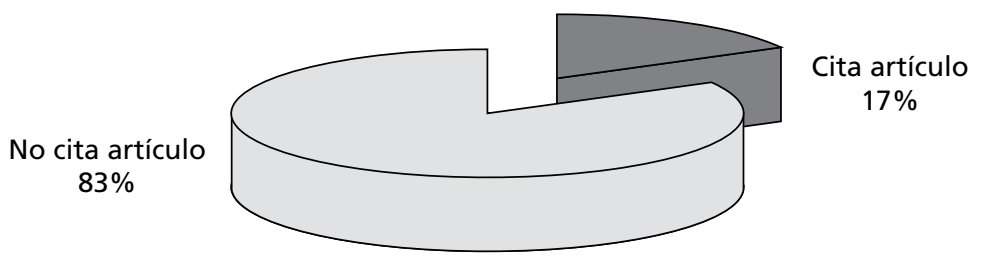

Fuente: Elaboración propia con información de la base de datos esp@cenet (2009).

\section{CONCLUSIONES Y FUTURAS LÍNEAS DE INVESTIGACIÓN}

La información examinada revela que el sector de las TIC español se sustenta en el conocimiento científico para desarrollar innovaciones potenciales en una proporción muy inferior al conocimiento tecnológico derivado de la tecnología patentada por la propia industria. Estos resultados abren varias líneas de reflexión sobre el diseño de las políticas científicas y tecnológicas nacionales y regionales, sobre todo de aquellas encaminadas a estimular la cooperación entre el ámbito científico y el tecnológico, políticas que favorezcan el desarrollo de la ciencia doméstica en campos científicos relacionados con sectores de cierta especialización regional o políticas de difusión que favorezcan el acceso de los sectores tecnológicos a la ciencia. A partir de la información obtenida en el análisis descriptivo podemos concluir de manera inicial que se confirman las hipótesis siguientes:

- Hipótesis 2: La nacionalidad de los inventores que intervienen en los procesos de innovación influye en el establecimiento de algún acuerdo de cooperación.

- Hipótesis 3: La incorporación de patentes como fuente externa de conocimientos para la innovación tiene que ver con la participación en acuerdos de cooperación.

- Hipótesis 5: El uso de artículos científicos como fuente externa de conocimientos para la innovación influye en el establecimiento de acuerdos de cooperación.

Es decir, que se ha detectado que cuando las empresas han innovado conjuntamente, existe mayor propensión a la incorporación de inventores extranjeros. Asimismo, que la incorporación de fuentes externas de conocimiento en forma de patentes y artículos científicos cobra mayor importancia como fruto de la colaboración que en el caso de que se presenta una sola empresa solicitante de la patente. De esta forma, los resultados nos llevan a rechazar las hipótesis 1 y 4 , por lo que se concluye que la elección del socio y el conteo de los distintos países de origen de las patentes citadas no es un factor determinante en el establecimiento de acuerdos de colaboración en procesos abiertos de innovación.

En cualquier caso, debe tenerse en cuenta que la interpretación de los flujos ciencia-tecnología efectuada en este trabajo se restringe al conocimiento codificado implícito en los datos recogidos en las patentes en un sector determi- 
nado. Deben entenderse, por tanto, como un punto de vista que motive una reflexión sobre las distintas facetas implicadas en el proceso de innovación.

De forma complementaria a la interpretación de los resultados, podemos concluir con base en la revisión de la bibliografía, que las organizaciones han comprendido que una forma de ser competitivas en áreas donde del dinamismo del cambio tecnológico es muy rápido o complejo, consiste en ser capaces de identificar los conocimientos requeridos en cualquier lugar donde existan, implantando un modelo basado en procesos abiertos en lugar de un modelo de innovación interna. Esta aproximación ha contribuido a un incremento sensible de la cooperación durante la última década debido a que la industria ha identificado grupos de investigación en universidades e instituciones y centros de investigación, como socios a tener en cuenta en este modelo.

Con estos elementos, podemos concluir la primera parte de nuestro estudio, que se refiere a la adquisición de conocimiento en procesos abiertos de innovación. Sin embargo, aún resta por explorar lo referente a las estrategias de apropiación y protección de la tecnología. Si bien a partir de la revisión de la bibliografía hemos situado estos elementos como parte fundamental en los procesos abiertos para la innovación, no ha sido posible detectar información en los datos incluidos en las patentes. Sin embargo, existe otra herramienta por la cual podemos acudir a las fuentes primarias de información, que para nuestra investigación son las empresas. Para ello, se propone la utilización del método de estudio de casos por constituir una herramienta valiosa de investigación, y su mayor fortaleza radica en que a partir de éste se mide y registra la conducta de las personas involucradas en el fenómeno estudiado (Yin, 1989). Además, en el método de estudio de caso los datos pueden ser obtenidos desde una variedad de fuentes tanto cualitativas como cuantitativas. Una vez obtenida esta información podremos completar nuestro objeto de análisis y emitir ciertas conclusiones globales sobre nuestro tema de investigación.

\section{REFERENCIAS BIBLIOGRÁFICAS}

Aho, E., J.Cornu, L. Georghiou, y A. Subirá (2006), Creating an innovative Europe. Report of the independent expert group on $R \& D$ and innovation, Bélgica, European Communities.

Álvarez, I., A. Fonfría y R. Marín (2005), "The role of networking in the competitiveness profiles of Spanish firms", Working Paper núm. 4, ICEI, UCM.

ASIMELEC (2009), Informe 2009 del Sector TIC en España, Madrid, Asociación Multisectorial de Empresas Españolas de Electrónica y Comunicaciones.

Cassiman, B. (1999), "Cooperación en investigación y desarrollo: Evidencia para la industria manufacturera española", Papeles de Economía Española, núm. 81, pp. $143-154$.

Cassiolato, J. (1994), “Innovación y cambio tecnológico", en E. Martínez (comp.), Ciencia, tecnología y desarrollo, Nueva Sociedad, Santiago, pp. 261-305.

Castells, M. (1996), La era de la información: economía sociedad y cultura. La sociedad red, Madrid, Alianza Editorial, Vol. I.

Chesbrough, H. (2003), Open Innovation: The New Imperative for Creating and Profiting from Technology, Boston, Harvard Business School Press.

(2006), "Open Innovation: A New Paradigm for Understanding Industrial Innovation", en H. Chesbrough, W. Vanhaverbeke y J. West (comps.), Open Innovation: Researching a New Paradigm, Nueva York, Oxford University Press, pp. 1-12.

Cohen, W., y D. Levinthal (1990), “Absorptive capacity: a new perspective on learning and innovation”, Administrative Science Quarterly, 35 (1), pp. 128-152.

COTEC (2000), "La innovación en las tecnologías de la información y las comunicaciones", Informes COTEC sobre el sistema español de innovación, Madrid, Fundación COTEC para la Innovación Tecnológica.

Dosi, G., y F. Malerba (1996), "Organizational learning and institutional embeddedness", en G. Dosi y F. Malerba (comps.), Organization and Strategy in the Evolution of Enterprise, Londres, Macmillan, pp. 1-16.

Etzkowitz, H., y L. Leydesdorff (1995), “The Triple Helix of University-Industry-Government Relations: A Laboratory for Knowledge Based Economic Development", EASST Review 14 (1), pp. 11-19.

, A. Webster, C. Gebhardt y B. Cantisano (2000), "The future of the university and the university of the future: evolution of ivory tower to entrepreneurial paradigm", Research Policy, 29, pp. 313-330.

Foray, D. (2004), The Economics of Knowledge, Cambridge, MIT Press. 
Freeman, Ch. (1995), "The National System of Innovation in Historical Perspective", Cambridge Journal of Economics, 19 (1), pp. 5-24.

García, E. (1992), "La cooperación interempresarial en España: Características de los acuerdos suscritos entre 1986 y 1989", Economía Industrial, julio-agosto: 113122.

Graves, A. (1987), "Comparative Trends in automotive Research and Development", DRC Discussion Paper, núm. 54, Science Policy Research Unit, Sussex University. Tomado de Rothwell (1994).

Guellec, D., y B. van Pottelsberghe (2000), "Applications, grants and the value of patent”, Economics Letters, 69 (1), pp. 109-114.

Handy, C. (1990), The age of unreason, Cambridge, Harvard Business School Press, Tomado de P. Morcillo (2006).

Hidalgo, A. (2003), Los patrones de innovación en España a través del análisis de patentes. Un análisis cualitativo del periodo 1988-1998, Madrid, Oficina Española de Patentes y Marcas.

Katila, R. (2002), "New product search over time: past ideas in their prime?", Academy of Management Journal, núm. 45, pp. 995-1010.

_ y G. Ahuja (2002), "Something old, something new: a longitudinal study of search behaviour and new product introduction", Academy of Management Journal, núm. 45, pp. 1183-1194.

Kline, S., y N. Rosenberg (1986), “An overview of innovation”, en R. Landau y N. Rosenberg (comps.), The positive sum strateg, Washington, National Academy Press, pp. 275-305.

Koput, K. (1997), "A chaotic model of innovative search: some answers, many questions", Organization Science, 8 (5), pp. 528-542.

Laursen, K., y A. Salter (2006), "Open for innovation: the role of openness in explaining innovation performance among U.K. manufacturing firms", Strategic Management Journal Vol. 27 (2), pp. 131-150

Leadbeater, Ch. (2006), Living on thin air, Penguin Books, Harmondsworth.

León, G. (2007), Evolución de las politicas de investigación e innovación de la Unión Europea y su impacto en España, Madrid, Universidad Politécnica de Madrid.

Lundvall, B. (comp.) (1992), National systems of innovation: towards a theory of innovation and interactive learning, Londres, Printer Publishers.

Menguzzato, M. (1992), "La cooperación: Una alternativa para la empresa de los 90", Dirección y Organización, núm. 4, pp. 54-62.

Metcalfe, S. (1995), “The Economic Foundations of Technology Policy: Equilibrium and Evolutionary Perspectives", en P. Stoneman (comp.), Handbook of the Econo- mics of Innovation and Technological Change, Oxford, Blackwell Publishers, pp. 513-557.

Mintzberg, H. (1983), The power in and around organization, Englewood, Prentice Hall.

Molero, J., y A. Hidalgo (2003), "Los sectores de alta tecnología en la Comunidad de Madrid”, en J. García (dir.), Estructura económica de Madrid, Madrid, Civitas, pp. 441-468.

Morcillo, P. (2006), Cultura e innovación empresarial. La conexión perfecta, Madrid, Thomson Editores.

Mowery, D., y N. Rosenberg (1978), "The Influence of Market Demand upon Innovation: a Critical Review of some recent Empirical Studies", Research Policy, 8 , pp. 102-153.

Nelson, R. (comp.) (1993), National Innovation Systems. A Comparative Analysis, Nueva York, Oxford University Press.

Nonaka, I., y H. Takeuchi (1995), The Knowledge Creating Company, Nueva York, Oxford University Press.

OCDE (1994), The measurement of scientific and technological activities: using patent dated as science and technology indicators. Patent Manual 1994.

(2005a), Compendium of patent statistics 2005.

(2005b), Oslo Manual $3^{\text {rd }}$. edition Guidelines for Collecting and Interpreting Innovation Data, París, Publishing.

Ohmae, K. (1991), El poder de la triada: las nuevas reglas de la competencia mundial. Madrid, McGraw Hill.

Porter, M. (1998), "Clusters and the new economics of competition", Harvard Business Review 76 (6), pp. 77-90.

Powell, W., K. Koput, L. Smith-Doerr y J. Owen-Smith (1999), "Network Position and Firm Performance: Organizational Returns to Collaboration in the Biotechnology Industry", Research in the Sociology of Organizations, núm.16, pp. 129159.

Rosenberg, N. (1982), Inside the Black Box: Technology and Economics, Nueva York, Cambridge University Press.

Rothwell, R. (1992), "Successful Industrial Innovation: Critical Factors for the 1990's", $R \& D$ Management, 22 (3), pp. 221-239.

Rozga, R. (1999), Entre globalización tecnológica y contexto nacional y regional de la innovación, un aporte a la discusión de la importancia de lo global y lo local para la innovación tecnológica, Toluca, V Seminario Internacional. 
Saxenian, A. (1994), Regional advance, culture and competition in Silicon Valley and Route 128, Cambridge, Harvard University Press,.

Schmookler, J. (1962), “Economic Sources of Inventive Activity", Journal of Ecomomic History. XXII (1), pp. 1-20.

Schumpeter, J. (1912), Theorie der wirtschaftlichen Entwicklung, Berlín, Duncker \& Humblodt.

(1934), The theory of economic development, Harvard University Press, Cambridge, Massachusetts.

(1942), Capitalismo, socialismo y democracia, J. Díaz (trad.), Madrid, Aguilar.

Scott, C.; Brown, J. (1999), "Bridging epistemologies: The generative dance between organizational knowledge and organizational knowing". Organization Science 10, pp. 381-400.

Shapiro, C., y H. Varian, (1998), Information rules: a strategic guide to the network economy, Harvard Business School Press, Boston, Massachusetts.

Stuart, T., y J. Podolny (1996), "Local search and the evolution of technological capabilities”, Strategic Management Journal, núm. 17, pp. 21-38.

Teece, D. (1986), "Profiting from technological innovation: implications for integration, collaboration, licensing and public policy", Research Policy, 15(6), pp. 285 305.

(1989), "Interorganizational requirements of the innovation process", Managerial and Decision Economics, 10 (1), pp. 35-42.

Villaseca, J., y J. Torrent (2008), “TIC, conocimiento y crecimiento económico. Un análisis empírico, agregado e internacional, sobre las fuentes de la productividad", Economía Industrial, 360, pp. 41-60.

Von Hippel, E. (1986), "Lead Users: A source of novel product concepts", Management Science, 32 (7), pp. 791-805.

West, J., y S. Gallager, (2006), "Open Innovation: A New Paradigm for Understanding Industrial Innovation”, en H. Chesbrough; W. Vanhaverbeke; J. West (comps.), Open Innovation: Researching a New Paradigm, Nueva York, Oxford University Press, pp 82-106.

Yin, R. (2003), Case Study Research: Design and Methods, California, Sage.

\section{A Microeconometric Analysis on the Pension Reforms in Argentina, Bolivia, Mexico and Uruguay}

Roberto Gallardo del Ángel*

\section{AbSTRACT}

This paper contains information about workers' participation in the pension schemes in four Latin American countries which carried out structural pension reforms in 1990's. I surveys some interesting hypotheses about the main causes that discourage participation in the new pension scheme. The main finding is that the present participation structure is strongly influenced by labor market characteristics like low-productivity and informal jobs.

Classification number: JEL: J26, J32.

Key words: pension reform, substitutive reforms, mixed reforms, private pensions, retirement policies.

\section{RESUMEN}

Este artículo contiene información sobre la participación de los trabajadores en el sistema de pensiones en cuatro países de América Latina, en los que las pensiones experimentaron reformas estructurales en 1990. Analiza algunas hipótesis interesantes sobre las principales causas que desalientan la participación de los trabajadores en el nuevo régimen de pensiones. El principal hallazgo es que la estructura de la participación actual está fuertemente influida por las características del mercado laboral, así como por la baja productividad y el empleo informal.

Número de clasificación: JEL: J26, J32.

Palabras clave: reformas en las pensiones, reformas sustitutivas, reformas mixtas, pensiones privadas, políticas de jubilación.
* Profesor de la Facultad de Economía de la Universidad Veracruzana. Correo electrónico: rogallardo@uv.mx. 